\title{
Four Testable Predictions of Instanton Cosmology
}

\author{
R.E. Allen \\ Center for Theoretical Physics, Texas A\&M University \\ College Station, Texas 77843, USA
}

\begin{abstract}
A new cosmological model makes the following predictions: (1) The deceleration parameter $q_{0}$ is approximately zero. (2) The mass density parameter $\Omega_{m}$ is less than 1. (3) The universe is spatially closed, but is asymptotically flat as $t \rightarrow \infty$, regardless of its matter content. (4) The age of the universe is approximately $15 \mathrm{Gyr}$ if the Hubble parameter $h$ is approximately 0.65.
\end{abstract}

In a new theory [1], the evolution of the cosmic scale factor $R(t)$ is determined by both the Einstein field equations and an $\mathrm{SU}(2)$ cosmological instanton. The instanton dominates in the later universe, making $R(t)$ approximately proportional to $t$ :

$$
d R / d t=K \quad, \quad t \rightarrow \infty
$$

where $K$ is a constant. On the other hand, Einstein gravity dominates in the early universe, giving the usual Friedmann equation

$$
(d R / d t)^{2}+k=(8 \pi G / 3) \rho R^{2} \quad, \quad t \rightarrow 0
$$

with $k$ positive (since the universe is spatially a 3 -sphere). Suppose that a crossover time $t_{c}$ is defined by

$$
(8 \pi G / 3) \rho R^{2}=K^{2}+k \quad, \quad t=t_{c} .
$$

Since $\rho R^{2}$ decreases with $R$ and $t$, we then have

$$
\rho<\left(\frac{3}{8 \pi G}\right) \frac{K^{2}+k}{R^{2}}<\left(\frac{3}{8 \pi G}\right)\left(\frac{d R / d t}{R}\right)^{2} \quad, \quad t \gg t_{c}
$$

or

$$
\Omega_{m}<1 \quad, \quad t \gg t_{c}
$$

where 


$$
\Omega_{m} \equiv \frac{\rho}{\rho_{c}} \quad, \quad \rho_{c} \equiv\left(\frac{3}{8 \pi G}\right) H^{2} \quad, \quad H \equiv \frac{d R / d t}{R} .
$$

According to (1), we also have

$$
q \equiv-\frac{d^{2} R / d t^{2}}{H^{2} R} \approx 0 \quad, \quad t \gg t_{c}
$$

With the reasonable assumption that the age of the universe is substantially larger than $t_{c}$, the present theory thus makes several testable predictions:

(1) The current deceleration parameter qo is approximately zero.

(2) The current mass density parameter $\Omega_{m}$ is less than 1 .

Two additional predictions are obtained below:

(3) The universe is spatially closed, but is asymptotically flat as $\mathbf{t} \rightarrow \infty$, regardless of its matter content.

(4) The age of the universe is approximately 15 Gyr if the Hubble parameter $h$ is approximately 0.65 .

Let us consider the origin of these results in more detail, beginning with the epoch $t \gg t_{c}$ where the cosmological instanton dominates. In the theory of Ref. 1 , this instanton is a topological defect in a GUT Higgs field which condenses in the very early universe. Its order parameter $\Psi_{s}$ has the symmetry group $\mathrm{SU}(2) \times \mathrm{U}(1) \times$ $\mathrm{SO}(10)$. The $\mathrm{SO}(10)$ gauge symmetry can be ignored in the present context, however, so $\Psi_{s}$ may be regarded as simply an $\mathrm{SU}(2) \times \mathrm{U}(1)$ order parameter in fourdimensional spacetime. With $x^{0}$ chosen to be the radial coordinate, there is a singularity at $x^{0}=0$ which is interpreted as the big bang. This singularity is physically admissible because it is perfectly analogous to the singularity at the center of a superfluid vortex. One can in fact define a "superfluid velocity"

$$
v^{\mu}=-i m^{-1} U^{-1} \partial^{\mu} U \quad \text { where } \quad \Psi_{s}=n_{s}^{1 / 2} U \eta_{0}
$$

and $\eta_{0}$ is a constant 2-component vector. The field $e_{\alpha}^{\mu}$ defined by

$$
e_{\alpha}^{\mu}=v_{\alpha}^{\mu} \quad, \quad v^{\mu}=v_{\alpha}^{\mu} \sigma^{\alpha}
$$

is interpreted as the vierbein which determines the geometry of spacetime [1].

If $a$ is the strength of the cosmological instanton centered on $x^{0}=0$, the constraint of fixed $\mathrm{SU}(2)$ topological charge requires that [1-3]

$$
e_{\alpha}^{\mu}=\delta_{\alpha}^{\mu} a / m x^{0} \quad, \quad e_{\mu}^{\alpha}=\delta_{\mu}^{\alpha} m x^{0} / a
$$


In addition, larger values of $x^{0}$ correspond to larger 3 -spheres [1], so $d x^{k}=x^{0} d \bar{x}^{k}$, where $d \bar{x}^{k}$ represents a distance within the unit 3 -sphere. There are thus two contributions to the expansion of the universe in the present model, with both the 3-space metric tensor $g_{k l}$ and the separation of comoving points increasing as functions of $x^{0}$.

The vierbein of (10) leads to a metric with the Robertson-Walker form

$$
\begin{aligned}
d s^{2} & =\eta_{\alpha \beta} e_{\mu}^{\alpha} e_{\nu}^{\beta} d x^{\mu} d x^{\nu} \\
& =-\left(\frac{m x^{0}}{a}\right)^{2}\left(d x^{0}\right)^{2}+\left[\frac{m\left(x^{0}\right)^{2}}{a}\right]^{2} d \bar{x}^{k} d \bar{x}^{k} \\
& =-d t^{2}+R(t)^{2}\left(\frac{d r^{2}}{1-r^{2}}+r^{2} d \Omega\right)
\end{aligned}
$$

where $\eta_{\alpha \beta}=\operatorname{diag}(-1,1,1,1)$ is the Minkowski metric tensor and

$$
R(t)=(m / a)\left(x^{0}\right)^{2} \quad, \quad d t=(m / 2 a) d\left(x^{0}\right)^{2} .
$$

Then (1) follows immediately, with $K=2$.

In the later universe, therefore, $R(t)$ is primarily determined by the topology of the cosmological instanton. The mass density $\rho$ has some influence at any finite time $t$, however, and the local metric tensor near a massive object is determined by the Einstein field equations to an extremely good approximation. As usual, these equations follow from the variational principle $\delta S_{L} / \delta g^{\mu \nu}=0$ if there are no constraints, where $S_{L}$ is the full Lorentzian action [1]. In the present theory, there is a constraint imposed by topology on a cosmological scale, but this constraint is of negligible importance on, e.g., a planetary scale. Near a dense concentration of mass in our highly heterogeneous universe, the local deformation of spacetime geometry is still accurately determined by the usual equations of general relativity.

Furthermore, the Einstein field equations are also a very good approximation in the early universe, since the instanton contribution of $(1)$ to $(d R / d t)^{2}$ is constant, whereas the usual gravitational contribution of (2) grows as $R \rightarrow 0$, with $\rho \propto R^{-3}$ (in the matter-dominated era) or $R^{-4}$ (in the radiation-dominated era).

The spatial curvature associated with (13) is proportional to $R(t)^{-2}$ [4]. The present model thus requires a universe that is asymptotically flat, because the topology of the cosmological instanton requires that $R(t) \propto t$ for $t \rightarrow \infty$. This is true regardless of the value of $\Omega_{m}$. Observations of the cosmic microwave background and of large scale structure indicate that the universe is very nearly flat but that $\Omega_{m} \approx 0.3[5]$.

For $t \gg t_{c},(1)$ implies that $H^{-1} \approx t$ rather than $\frac{3}{2} t$. The age of the universe is then given by $t_{0} \approx H_{0}^{-1}$ rather than $\frac{2}{3} H_{0}^{-1}$. With the conventional definition 
$H_{0}=100 h \mathrm{~km} \mathrm{~s}^{-1} \mathrm{Mpc}^{-1}$, a value of $h=0.65[6,7]$ implies that $t_{0} \approx 15$ Gyr rather than 10 Gyr. This larger value is more consistent with recent estimates of $10-14$ Gyr for the ages of the oldest globular clusters [8].

The present theory thus makes a number of testable predictions. In particular, the prediction $q_{0} \approx 0[9]$ clearly demarcates the present theory from standard cosmology with a flat spacetime, in which $q_{0}=1 / 2[10,11]$, and from $\Lambda$ CDM models, in which $q_{0} \approx-1 / 2[12]$. Quantitative measurements of $q_{0}$ are now becoming attainable $[13,14]$, so it should be possible to subject this prediction to convincing tests in the near future.

\section{Acknowledgement}

This work was supported by the Robert A. Welch Foundation.

\section{REFERENCES}

1. R.E. Allen, Int. J. Mod. Phys. A 12, 2385 (1997), hep-th/9612041.

2. R.E. Allen, proceedings of the CPT-98 Meeting on CPT and Lorentz Symmetry, edited by V.A. Kostelecký (World Scientific, Singapore, 1999); hep-ph/9902228.

3 . The $e_{\mu}^{\alpha}$ are "ordinary" components, after the initial coordinates of Ref. 1 are transformed to four-dimensional spherical coordinates. See S. Weinberg, Gravitation and Cosmology (Wiley, New York, 1972), p. 109.

4. C.W. Misner, K.S. Thorne, and J.A. Wheeler, Gravitation (W.H. Freeman, San Francisco, 1973)

5. M. Webster, M.P. Hobson, A.N. Lasenby, O. Lahav, and G. Rocha, astro$\mathrm{ph} / 9802109$, and references therein.

6. D. Branch, Ann. Rev. of Astronomy and Astrophysics 36, 17 (1998), astro$\mathrm{ph} / 9801065$, and references therein.

7. W. L. Freedman, J. R. Mould, R. C. Kennicutt, and B. F. Madore, astro-ph/9801080, and references therein.

8. See, for example, B. Chaboyer, P. Demarque, P. J. Kernan, and L. M. Krauss, Astrophys. J. 494, 96 (1998); astro-ph/9706128.

9. There have been other "coasting universe" proposals, but they involve exotic forms of matter or other ad hoc assumptions, whereas here the result $q_{0} \rightarrow 0$ as $t \rightarrow \infty$ emerges automatically within the context of a more general theory. See, e.g., E.W. Kolb, Astrophys. J. 344, 543 (1989); M. Kamionkowski and N. Toumbas, Phys. Rev. Lett. 77, 587 (1996); M. Özer and M.O. Taha, Nuclear Physics B 287, 776 (1987).

10. P.J.E. Peebles, Principles of Physical Cosmology (Princeton University Press, Princeton, 1993).

11. E.W. Kolb and M.S. Turner, The Early Universe (Addison-Wesley, Redwood City, California, 1990).

12. M.S. Turner, Nucl. Phys. A621, 522c (1997); astro-ph/9704024.

13. S. Perlmutter et al., Nature 391, 51 (1998); astro-ph/9712212.

14. P. M. Garnavich et al., Astrophys. J. 493, L53 (1998); astro-ph/9710123. 